PACS 31.15.NE, 31.10.1z

A. O. Makarova, A. A. Buyadzhi, O. V. Dubrovsky

\author{
Odessa National Maritime Academy, Odessa, 4, Didrikhsona str., Odessa, Ukraine \\ e-mail: buyadzhiaa@gmail.com
}

\title{
SPECTROSCOPY AND DYNAMICS OF MULTIELECTRON ATOM IN A MAGNETIC FIELD: NEW APPROACH
}

\begin{abstract}
Spectroscopy and dynamics of multielectron atomic system in a magnetic field is numerically investigated. It is presented a new quantum-mechanical approach to calculating the energies and widths of some states for the multielectron atomic system in in a homogeneous magnetic field. The approach is based on the numerical difference solution of the Schrödinger equation, the model potential method and the operator perturbation theory formalism. As illustration, the data for energies of the electronic excited and ground state of the lithium atom in dependence upon the magnetic field strength are listed and compared with available theoretical results, obtained on the basis of alternative Hartree-Fock method.
\end{abstract}

\section{Introduction}

The hydrogen atom in a constant magnetic field has been considered in a fairly large number of studies, however, many of the results turn out to be either unsuitable for specific applications or even incomplete until recently (see, for example, [1-5]). In the case of many-electron (non-hydrogen-like) atomic systems in a magnetic field, the situation looks dramatic enough. The fact is that generalizing the model to the case of many-electron atoms is quite problematic. Traditional methods such as perturbation theory, models based on asymptotic expansions in the magnitude of the field $\mathrm{B}$, quasiclassical approaches (see, [1-14]) encounter significant problems when generalizing to the case of many-electron systems. Particularly acute is the problem of describing the dynamics of an atomic system in the intermediate region of magnetic field strengths, where it is necessary to consider the Coulomb and magnetic interactions on an equal footing. The problem is also relevant for the field of strong and superstrong fields, where today there are no sufficiently reliable data on the energy characteristics of atomic systems in the field. On the whole, at present, sufficiently convenient universal data for arbitrary states of many-electron atomic systems are absent for any values of the magnetic field B. Among modern methods for describing atomic spectroscopy in a magnetic field, a series of papers [4-18] should be distinguished, where perturbation theory methods, various schemes, and algorithms have been developed based on the numerical solution of the Schrödinger equation in the Hartree-Fock and other approximations. Based on them, it was possible to obtain a lot of useful numerical data regarding the energies of various states of a number of many-electron atoms at various magnetic field intensities. At the same time, in a number of cases, as the authors admit $[3,8]$, their data require clarification due to the neglect of correlation effects, relativistic corrections, and other factors. Also relevant is the problem of describing the stochastic behavior of an atomic system in a magnetic field. It should be noted that various aspects of stochasticity in systems and the main features of quantum chaos that take place in the dynamics of many-electron atomic systems of atomic systems in a magnetic field are currently either partially or completely not studied, at least at a detailed quantitative level [14]. Naturally, therefore, the solution of the problem of a quantitative description of the elements of quantum chaos in the behavior of many-electron atomic systems in a static magnetic field seems extremely urgent and quite complicated (see [2]).

In this paper we shortly present a new quantum-mechanical approach to calculating the energies and widths of some states for the multielectron atomic system in in a homogeneous magnetic field. The approach is based on the numerical difference solution of the Schrödinger equation, the model potential method and the operator perturbation theory formalism. 


\section{Theoretical approach}

The Hamiltonian of a multielectron atom in a magnetic field B differs from the operator of the hydrogen atom by the presence of the Coulomb interaction operator, which naturally aggravates the problem of separation of variables in the Schrödinger equation. Introducing a cylindrical coordinate system $(\rho, \varphi, z)$, with the axis $\mathrm{Oz} \|$ $\mathrm{B}$ and taking into account that the dependence of the wave function on the rotation angle $\varphi$ around the $\mathrm{z}$ axis is trivial:

$$
\Psi \sim e^{i M \varphi} \psi(\rho, z)
$$

one should write the Schrödinger equation for the one-electron function of an atomic system (atomic units are used here $e=h=m=1$ ) as:

$$
\begin{aligned}
& {\left[\frac{\partial^{2}}{\partial \rho^{2}}+\frac{1}{\rho} \frac{\partial}{\partial \rho}+\frac{\partial^{2}}{\partial z^{2}}-\frac{M^{2}}{\rho^{2}}-4 \gamma^{2} \rho^{2}+\right.} \\
& \left.+\frac{4}{r}+V_{c}(r)+\left(\frac{E}{R_{y}}-\gamma M\right)\right] \Psi(\rho, z)=0
\end{aligned}
$$

where $\mathrm{V}_{\mathrm{c}}(\mathrm{r})$ is the potential that describes the effect of all other electrons on the given one. Naturally, it is absent for the hydrogen atom. As the potential Vc, we use the Green-like potential (c.g.[2]), which approximates the Hartree potential quite accurately:

$$
V=-\frac{\left(N_{c}-1\right) \Omega(r)}{r}
$$

where function $\Omega(r)=1 /[H \exp (r / d-1)+1]$ is the shielding function and $\mathrm{H}, \mathrm{d}$ are the parameters of the potential. The required parameters, as a rule, are selected from the condition of the best fitting of the experimental values of the energy levels of free atoms (c.g. [2]). Note that a potential of type (2) was used intensively in calculating the energy levels and oscillator strengths of various atomic systems, including $\mathrm{Li}, \mathrm{Be}, \mathrm{B}$, $\mathrm{C}, \mathrm{N}, \mathrm{O}, \mathrm{F}, \mathrm{Ne}$, and others (see [17-20]). To take into account the exchange corrections, the exchange potential was taken in the simplest Slater approximation and added to potential (3) [19]. The two-dimensional equation (2) is naturally not solved analytically in a general form. The terms appearing in it: the potential of the Coulomb interaction, which contains $r=\left(\rho^{2}+z^{2}\right)^{1 / 2}$, potential V $\left[\left(\rho^{2}+z^{2}\right)^{1 / 2}\right]$ prevents the separation of variables. One could rewrite the Schrödinger equation as follows:

$$
\begin{gathered}
H \psi(\rho, z)=E \psi(\rho, z) \\
H=-1 / 2\left(\partial^{2} / \partial \rho^{2}+1 / \rho \partial / \partial \rho+\right. \\
\left.+\partial^{2} / \partial z^{2}-m^{2} / \rho^{2}\right), V(\rho, z), \\
V(\rho, z)=-\left(\rho^{2}+z^{2}\right)^{-1 / 2}+\left(N_{c}-1\right) . \\
\Omega\left(\rho^{2}+z^{2}\right)^{-1 / 2}+1 / 8 \gamma^{2} \rho^{2}+m / 2,
\end{gathered}
$$

The potential $1 / 8 \gamma^{2} \rho^{2}$ limits the movement in the direction perpendicular to the field direction. Similarly, in the region $\gamma>>1$, the motion of an electron across a magnetic field is determined by the size of its cyclotron orbit, $\lambda=(\hbar c / e M)^{1 / 2}$ and along the field by a modified Coulomb interaction, which takes into account the non-Coulomb character of the potential field in which an electron moves in a many-electron atom [18]. Note that calculations of multielectron atomic systems with introduced potentials are quite well known in the literature (see $[2,18$ 20]); moreover, computational schemes based on them have been tested several times and tested for a number of atoms in the free state. The potential (3) was successfully used in calculating the energies and forces of atomic oscillators of the $1 \mathrm{st}$ period of the periodic table (see review in [21]). For solution of the Schrödinger equation with hamiltonian equations (7) we constructed the finite differences scheme which is in some aspects similar to method [2]. An infinite region is exchanged by a rectangular region: $0<\rho<L_{\rho}$, $0<\mathrm{z}<L_{Z}$. It has sufficiently large size; inside it a rectangular uniform grid with steps $h_{\rho}, h_{z}$ was constructed. The external boundary condition, as usually, is: $(\partial \Psi \partial n)_{r}=0$. The knowledge of the asymptotic behaviour of wave function in the infinity allows to get numeral estimates for $L_{\rho}, L_{Z}$. A wave function has an asymptotic of the kind as: $\exp \left[-(-2 E)^{1 / 2} r\right]$, where $(-E)$ is the ionization energy from stationary state to lowest Landau level. Then $L$ is estimated as $L \sim 9(-$ $2 E)^{-1 / 2}$. The more exact estimate is found empirically. The three-point symmetric differences scheme is used for second derivative on $\mathrm{z}$. The derivatives on $\square$ are approximated by $(2 m+1)$ point symmetric differences scheme with the use of the Lagrange interpolation formula differentiation. To calculate the values of the width $\mathrm{G}$ for resonances in atomic spectra in a magnetic field one can use the modified operator perturba- 
tion theory method (see details in Ref. $[12,13]$ ). Note that the imaginary part of the state energy in the lowest PT order is:

$$
\text { fm } E=G / 2=\pi<\Psi_{B}|H| \Psi_{E}>^{2}
$$

with the total Hamiltonian of system in a magnetic field. The state functions $\Psi_{\mathrm{Eb}}$ and $\Psi_{\mathrm{Es}}$ are assumed to be normalized to unity and by the $\delta(\mathrm{k}-\mathrm{k}$ ')-condition, accordingly. Other calculation details can be found in Refs. [2, 19-21].

\section{Illustration results and conclusion}

As illustration, below we present the data (tables 1 and 2) for energies of the electronic excited and ground state of the lithium atom in dependence upon the magnetic field strength (parameter $\gamma$ ) and compared with available theoretical results, obtained on the basis of alternative methods. Parameter $\gamma$ varies within: $\gamma=B$ / $B o=0.00-10$, where $B_{0}=m^{2} e^{3} c / h^{3} Z^{3}$. In Table 1 there are listed the energies of the ground state of the lithium atom in dependence upon the parameter $\gamma$. For the lithium atom there are available the results of calculations for the ground state and a few low-lying states of the $\mathrm{Li}$ atom at the regime of weak and intermediate fields. In particular, the Hartree-Fock (HF) calculation results are in the Refs. [6,7]. As the ground state analysis shows, in whole our data are corresponding to the alternative HF results, however, indeed, they lie a little lower for a weak field regime and more substantially lower in the intermediate regime of the magnetic parameter. In table 2 similar data are listed for the Li excited state.

Table 1.

Total energies (in a.u.) of the ground state of Li atom in a magnetic field with strength $\gamma$ : HF-mesh- the Hartree-Fock data from [6], HF - data from [7], MP- this work;

\begin{tabular}{|c|c|c|c|}
\hline$\gamma$ & $\begin{array}{c}1 \mathrm{~s}^{2} 2 \mathrm{~s} \\
\text { HF-mesh }\end{array}$ & $\begin{array}{c}1 \mathrm{~s}^{2} 2 \mathrm{~s} \\
\mathrm{HF}\end{array}$ & $\begin{array}{c}1 \mathrm{~s}^{2} 2 \mathrm{~s} \\
\mathrm{MP}\end{array}$ \\
\hline 0.000 & 27.4328 & 27.4327 & 27.4329 \\
\hline 0.002 & 27.4338 & & 27.4340 \\
\hline 0.009 & 27.4371 & 27.4371 & 27.4373 \\
\hline 0.020 & 27.4421 & & 27.4424 \\
\hline
\end{tabular}

\begin{tabular}{|l|l|l|l|}
\hline 0.126 & 27.4741 & 27.4739 & 27.4745 \\
\hline 0.200 & 27.4840 & & 27.4843 \\
\hline 0.900 & 27.4250 & 27.4240 & 27.4253 \\
\hline 1.800 & 27.2460 & 27.2446 & 27.2464 \\
\hline 2.000 & 27.1962 & & 27.1967 \\
\hline 2.500 & 27.0562 & & 27.0568 \\
\hline 3.600 & 26.6787 & 26.6640 & 26.6793 \\
\hline 5.000 & 26.0881 & & 26.0887 \\
\hline 5.400 & 25.9011 & 25.8772 & 25.9018 \\
\hline 7.000 & 25.0891 & & 25.0902 \\
\hline
\end{tabular}

Table 2.

Total energies (in a.u.) of the excited state of the Li atom in the magnetic field with the strength $\gamma$ : HF-mesh- the Hartree-Fock data from [6], HF - data from [7], MP- this work

\begin{tabular}{|l|l|l|l|}
\hline$\gamma$ & \multicolumn{1}{|c|}{$\begin{array}{c}1 \mathrm{~s}^{2} 2 \mathrm{p}_{-1} \\
\text { HF-mesh }\end{array}$} & $\begin{array}{c}1 \mathrm{~s}^{2} 2 \mathrm{p}_{-1} \\
\text { HF }\end{array}$ & \multicolumn{1}{|c|}{$\begin{array}{c}1 \mathrm{~s}^{2} 2 \mathrm{p}_{-1} \\
\text { MP }\end{array}$} \\
\hline 0.000 & 27.3651 & 27.3651 & 27.3652 \\
\hline 0.002 & 27.3671 & & 27.3673 \\
\hline 0.009 & 27.3739 & 27.3738 & 27.3741 \\
\hline 0.020 & 27.3840 & & 27.3843 \\
\hline 0.126 & 27.4565 & 27.4565 & 27.4568 \\
\hline 0.200 & 27.4922 & & 27.4925 \\
\hline 0.900 & 27.6563 & 27.6563 & 27.6567 \\
\hline 1.800 & 27.6766 & 27.6747 & 27.6770 \\
\hline 2.000 & 27.6625 & & 27.6631 \\
\hline 2.500 & 27.6035 & & 27.6041 \\
\hline 3.600 & 27.3764 & 27.3627 & 27.3771 \\
\hline 5.000 & 26.9423 & & 26.9430 \\
\hline 5.400 & 26.7952 & 26.7747 & 26.7959 \\
\hline 7.000 & 26.1267 & & 26.1279 \\
\hline
\end{tabular}

The difference between the listed data can be explained by the partial account of electron correlation corrections, which is absent in the HF calculation. 


\section{References}

1. Lisitsa, V.S. New results on the Stark and Zeeman effects in the hydrogen atom. Sov. Phys. Usp. 1987, 30, 927-960.

2. Glushkov, A.V. Atom in an electromagnetic field. KNT: Kiev, 2005.

3. Ignatenko, A., Svinarenko A., Prepelitsa, G.P., Perelygina, T.B. Optical bi-stability effect for multi-photon absorption in atomic ensembles in a strong laser field. Photoelectronics. 2009, 18, 103-105.

4. Rao,J., Liu, W., Li, B. Theoretical complex Stark energies of hydrogen by a complexscaling plus B-spline approach. Phys. Rev. A. 1994, 50, 1916-1919.

5. Rao, J.; Li, B. Resonances of hydrogen atom in strong parallel magnetic and electric fields. Phys.Rev.A. 1995, 51, 4526

6. Ivanov, M.V., Schmelcher, P. Ground state of the lithium atom in strong magnetic fields. Phys.Rev.A. 1998, 57, 3793-3800.

7. Jones, M., Ortiz, G., Ceperley, D. Hartree-Fock studies of atoms in strong magnetic fields. Phys. Rev. A. 1996, 54, 219-231.

8. Gadiyak G., Lozovik Yu.E., Mashchenko A., Obrecht M. Hydrogen-like and helium-like systems in a superstrong magnetic field. Opt. Spectr. 1984, 56 (1), 26-32

9. Khetselius, O., Lopatkin Y., Dubrovskaya, Y., Svinarenko A. Sensing hyperfine-structure, electroweak interaction and parity non-conservation effect in heavy atoms and nuclei: New nuclear-QED approach. Sensor Electr. and Microsyst. Techn. 2010, 7(2), 11-19.

10. Glushkov, A., Malinovskaya, S, Ambrosov S., Shpinareva I, Troitskaya O. Resonances in quantum systems in strong external fields: consistent quantum approach. J. Techn.Phys. 1997, 38(2), 215-218.

11. Ambrosov S., Ignatenko V., Korchevsky D., Kozlovskaya V. Sensing stochasticity of atomic systems in crossed electric and magnetic fields by analysis of level statistics for continuous energy spectra. Sensor Electr. and Microsyst. Techn. 2005, Issue 2, 19-23.

12. Glushkov, A.; Ambrosov, S.; Ignatenko, A. Non-hydrogenic atoms and Wannier-Mott excitons in a DC electric field: Photoionization, Stark effect, Resonances in ionization continuum and stochasticity. Photoelectronics, 2001, 10, 103-106.
13. Glushkov A.V.; Ivanov, L.N. DC strong-field Stark effect: consistent quantum-mechanical approach.J. Phys. B: At. Mol. Opt. Phys. 1993, 26, L379-386.

14. Rusov V., Glushkov A., Vaschenko V., Korchevsky D., Ignatenko A. Stochastic dynamics of the atomic systems in the crossed electric and magnetic field: the rubidium atom recurrence spectra. Bull.of Kiev Nat. Univ. 2004, N4, 433.

15. Khetselius, O. Relativistic perturbation theory calculation of the hyperfine structure parameters for some heavy-element isotopes. Int. $J$. Quant.Chem. 2009, 109, 3330-3335.

16. Glushkov, A.; Gurskaya, M.; Ignatenko, A.; Smirnov, A.; Serga, I.; Svinarenko, A.; Ternovsky E. Computational code in atomic and nuclear quantum optics: Ad-vanced computing multiphoton resonance parameters for atoms in a strong laser field. J. Phys.: Conf. Ser. 2017, 905, 012004.

17. Buyadzhi, V., Zaichko, P., Antoshkina, O., Kulakli, T., Prepelitsa, P., Ternovsky, V., Mansarliysky, V. Computing of radiation parameters for atoms and multicharged ions within relativistic energy approach: Advanced Code. $J$. Phys.: Conf. Ser. 2017, 905(1), 012003.

18. Svinarenko, A., Glushkov, A., Khetselius, O., Ternovsky,V., Dubrovskaya, Yu., Kuznetsova, A., Buyadzhi, V. Theoretical spectroscopy of rare-earth elements: spectra and autoionization resonances. Rare Earth Element, InTech. 2017, 83-104

19. Glushkov, A., Khetselius, O., Svinarenko A.A., Buyadzhi, V.V., Ternovsky, V., Kuznetsova, A., Bashkarev, P. Relativistic perturbation theory formalism to computing spectra and radiation characteristics: application to heavy element. Recent Studies in Perturbation Theory. InTech. 2017, 131-150.

20. Glushkov A.V., Khetselius O.Yu., Svinarenko A.A., Buyadzhi V.V., Methods of computational mathematics and mathematical physics. TES: Odessa, 2015

21. Khetselius, O., Gurnitskaya, E., Loboda, A., Vitavetskaya, L. Consistent quantum approach to quarkony energy spectrum and semiconductor superatom and in external electric field Photoelectron. 2008, 17, 127. 


\title{
SPECTROSCOPY AND DYNAMICS OF MULTIELECTRON ATOM IN A MAGNETIC FIELD: NEW APPROACH
}

\begin{abstract}
Summary. Spectroscopy of multielectron atomic system in a magnetic field is numerically investigated. It is presented a new quantum approach to calculating energies and widths of states for multi-electron atomic system in a homogeneous magnetic field. The approach is based on numerical difference solution of the Schrödinger equation, model potential method and operator perturbation theory. The data for energies of electronic excited and ground state of the lithium atom in dependence upon the magnetic field strength are listed and compared with available theoretical results, obtained on the basis of alternative Hartree-Fock method.
\end{abstract}

Key words: atomic system, magnetic field, spectroscopy and dynamics

PACS 31.15.Ne, 31.10.1z

\author{
А. О Макарова, А. А. Буяджи, О. В. Дубровский
}

\section{СПЕКТРОСКОПИЯ И ДИНАМИКА МНОГОЭЛЕКТРОННОГО АТОМА В МАГНИТНОМ ПОЛЕ: НОВЫЙ ПОДХОД}

Резюме. Изучается спектроскопия многоэлектронных атомных систем в магнитном поле. Представлен новый квантовый подход к расчету энергий и ширин состояний для многоэлектронного атома в однородном магнитном поле. Метод основан на численном разностном решении уравнения Шредингера, методе модельного потенциала и операторной теории возмущений. Приведены расчетные данные для энергий основного и возбужденного состояний атома лития в зависимости от напряженности магнитного поля и проведено сравнение с имеющимися теоретическими результатами, полученными на основе альтернативного метода Хартри-Фока.

Ключевые слова: атомная система, магнитное поле, спектроскопия и динамика

PACS 31.15.Ne, 31.10.1z

\author{
О. О. Макарова, Г. А. Буяджи, О. В. Дубровський
}

\section{СПЕКТРОСКОПІЯ І ДИНАМІКА БАГАТОЕЛЕКТРОННОГО АТОМА У МАГНІТНОМУ ПОЛІ: НОВИЙ ПІДХІД}

Резюме. Вивчається спектроскопія багато електронних атомних систем в магнітному полі. Представлений новий квантовий підхід до розрахунку енергій і ширин станів для багатоелектронного атома в однорідному магнітному полі. Метод заснований на чисельному різницевому рішенні рівняння Шредінгера, методі модельного потенціалу та операторній теорії збурень. Наведені розрахункові дані для енергій основного та збудженого станів атома літію в залежності від напруженості магнітного поля і проведено порівняння з наявними теоретичними результатами, отриманими на основі альтернативного методу Хартрі-Фока.

Ключові слова: атомна система, магнітне поле, спектроскопія та динаміка 